doi: 10.2306/scienceasia1513-1874.2013.39.327

\title{
Uraria pseudoacuminata, a new species from Thailand
}

\author{
Worachat Tokaew $^{\mathrm{a}, *}$, Pranom Chantaranothai ${ }^{\mathrm{b}}$ \\ a Department of Biology, Faculty of Science and Technology, Rajabhat Mahasarakham University, \\ Maha Sarakham 44000 Thailand \\ b Applied Taxonomic Research Centre, Department of Biology, Faculty of Science, Khon Kaen University, \\ Khon Kaen 40002 Thailand
}

*Corresponding author, e-mail: tokaew9@gmail.com

Received 27 Jul 2012

Accepted 19 Mar 2013

\begin{abstract}
A new species of the genus Uraria, U. pseudoacuminata, is described from Thailand. It differs from $U$. acuminata in having shorter racemes, hooked hairs on the lower part of its pedicels, and glabrous ovaries with only 2 , rather than $4(5)$ ovules. It also tends to be shorter and have shorter leaves and leaflets. A detailed description and illustrations are provided.
\end{abstract}

KEYWORDS: Papilionoideae, Fabaceae, legume family

\section{INTRODUCTION}

The genus Uraria Desv. (Leguminosae-Papilionoideae) comprises approximately 20 species and has a centre of diversity in India to Indo-China ${ }^{1}$. The first attempt to enumerate the species in this genus was made by Craib \& $\mathrm{Kerr}^{2}$, who listed 11 species in Thailand. During the fieldwork and herbarium studies aimed at completing a revision of this genus for the Flora of Thailand, a new species was discovered which is described and illustrated here.

\section{MATERIALS AND METHODS}

\section{Morphological study}

Field collections and herbarium material were studied. The morphological characteristics of the specimens described herein as $U$. pseudoacuminata sp. nov. were compared with those of the similar species, U. acuminata Kurz. Voucher specimens are deposited at KKU. Additional materials have been examined and consulted from the following herbaria (abbreviations according to Ref. 3): AAU, BCU, BK, BKF, BM, C, CMU, K, KKU, P, PBM, PSU, QBG, TCD, and Herbarium of the Department of Biology, Chiang Mai University, Thailand.

\section{DESCRIPTION OF THE SPECIES}

Uraria pseudoacuminata W. Tokaew \& Chantar., sp. nov.- Figs. 1, 2c-e. Affinis Urariae acuminatae sed ovarium 2-ovulatum, legumina segmentis 2, glabris, pedicellis pilis inferioribus sparsis glandulis multicellularis et glochidiis raro rectis differt.- Typus: W. Tokaew \& P. Chantaranothai 403 (holotype KKU),

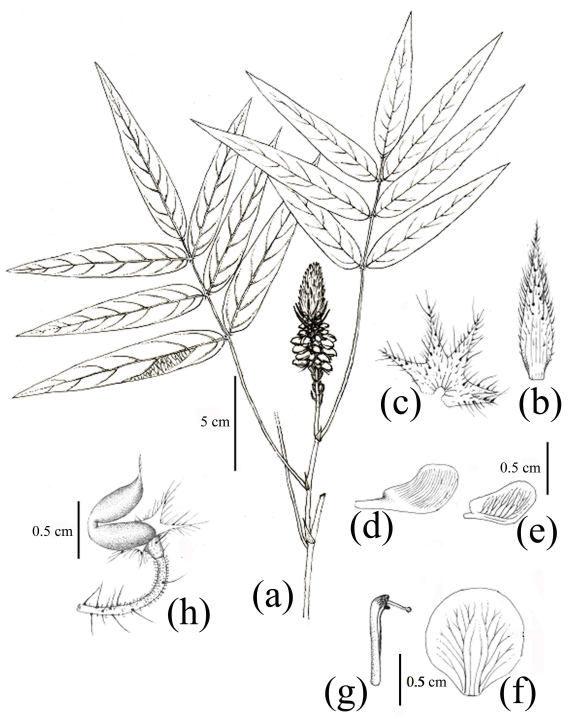

Fig. 1 Uraria pseudoacuminata: (a) habit, (b) bract, (c) calyx, (d) keel, (e) wing, (f) standard, (g) stamen and pistil, (h) fruit. (W. Tokaew \& P. Chantaranothai 403 (KKU)) Drawn by Worachat Tokaew.

Thailand, Bueng Kan province, Bungkhla, Phu Wua Wildlife Sanctuary, deciduous forest, alt. 240 m, 21 October 2007.

Unbranched shrubs $40-60 \mathrm{~cm}$ high. Leaves (3-) 5-7-foliolate; petioles 6-10 cm long, with both unicellular, hooked hairs and straight hairs; stipules deltoid, acuminate, $7-8$ by $2-3 \mathrm{~mm}$. Leaflets slightly coria- 


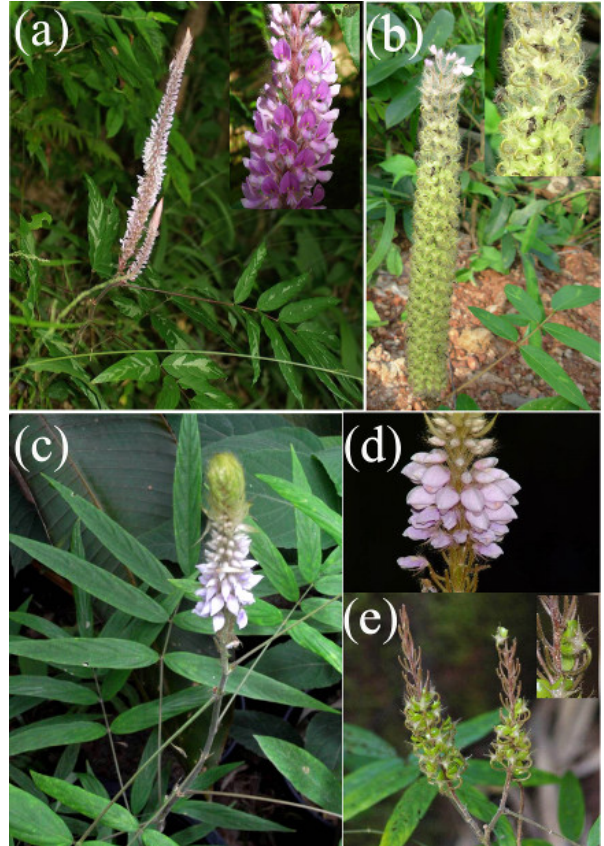

Fig. 2 Comparative morphological characters of Uraria acauminata and $U$. pseudoacauminata. $U$. acauminata (a) habit and inflorescence, (b) fruits; U. pseudoacauminata (c) habit and inflorescence, (d) flowers, (e) fruits.

ceous, bright green, lanceolate or narrowly lanceolate, $7-12$ by (1-) $2-3.5 \mathrm{~cm}$, base obtuse, apex acuminate, margin entire, upper surface glabrate, lower surface pubescent, both surfaces with both unicellular, hooked hairs and straight hairs; petiolules $1.5-2 \mathrm{~mm}$ long, with unicellular, straight hairs; stipels narrowly lanceolate, acuminate, $1.5-2$ by $1 \mathrm{~mm}$. Inflorescences racemose, terminal, erect, $10-15 \mathrm{~cm}$ long. Flowers densely packed on the axis; bracts lanceolate, 1214 by $1-2 \mathrm{~mm}$, pale purple or pale green; pedicels $8-10 \mathrm{~mm}$ long, with unicellular, hooked hairs and multicellular, glandular hairs, rarely with unicellular, straight hairs. Calyx pale purple, with unicellular, straight hairs and multicellular, glandular hairs; tube ca. $2 \mathrm{~mm}$ long; upper lobes 2, $c a .2 .5 \mathrm{~mm}$ long; lower lobes 3, thread-like, ca. $4 \mathrm{~mm}$ long. Corolla pale violet; standard $10-12$ by $9-10 \mathrm{~mm}$, claw ca. $2 \mathrm{~mm}$ long; wings $8-9$ by $3-3.5 \mathrm{~mm}$, claw ca. $1 \mathrm{~mm}$ long; keels $8-9$ by $3-3.5 \mathrm{~mm}$, claw $2-2.5 \mathrm{~mm}$ long. Ovary 2-ovuled. Legumes with 2 segments, each segment orbicular, $4-5$ by $3-4 \mathrm{~mm}$, connected on the transverse side of each segment, pale brown, glabrous. Seeds reniform, $2-3$ by $2 \mathrm{~mm}$, pale brown.

Distribution.- Thailand.

Habitat and ecology.- In dry evergreen and deciduous forests, alt. $300-1000$ m. Flowering August-
Table 1 Differences between Uraria acuminata and U. pseudoacuminata.

\begin{tabular}{|c|c|c|}
\hline Characters & U. acuminata & U. pseudoacuminata \\
\hline Height of shrub & $50-150 \mathrm{~cm}$ & $40-60 \mathrm{~cm}$ \\
\hline Leaflet number & (3-) 5-9 & $(3-)$ 5-7 \\
\hline Leaflet shape & $\begin{array}{l}\text { lanceolate, rarely } \\
\text { elliptic or narrowly } \\
\text { oblong }\end{array}$ & $\begin{array}{l}\text { lanceolate to nar- } \\
\text { rowly lanceolate }\end{array}$ \\
\hline Petiole length & $10-15 \mathrm{~cm}$ & $6-10 \mathrm{~cm}$ \\
\hline Petiolule length & $2-3(-4) \mathrm{mm}$ & $1.5-2 \mathrm{~mm}$ \\
\hline Raceme length & $25-40 \mathrm{~cm}$ long & $10-20 \mathrm{~cm}$ long \\
\hline Bract colour & $\begin{array}{l}\text { pale pink or pale } \\
\text { purple }\end{array}$ & $\begin{array}{l}\text { pale purple or pale } \\
\text { green }\end{array}$ \\
\hline Calyx colour & pale pink & pale purple \\
\hline Corolla colour & pink or purple & pale violet \\
\hline $\begin{array}{l}\text { Hairs on upper } \\
\text { part of pedicel }\end{array}$ & $\begin{array}{l}\text { unicellular hooked } \\
\text { hairs and rarely } \\
\text { multicellular } \\
\text { glandular hairs }\end{array}$ & $\begin{array}{l}\text { unicellular hooked } \\
\text { hairs and rarely } \\
\text { multicellular } \\
\text { glandular hairs }\end{array}$ \\
\hline $\begin{array}{l}\text { Hairs on lower } \\
\text { part of pedicel }\end{array}$ & $\begin{array}{l}\text { dense unicellular } \\
\text { straight hairs and } \\
\text { rarely multicellular } \\
\text { glandular hairs }\end{array}$ & $\begin{array}{l}\text { sparse multicellular } \\
\text { glandular hairs, } \\
\text { unicellular hooked } \\
\text { hairs, and rarely uni- } \\
\text { cellular straight hairs }\end{array}$ \\
\hline Ovule number & $4(-5)$ & 2 \\
\hline Pod segments & $\begin{array}{l}3-4 \text {, with straight } \\
\text { hairs }\end{array}$ & 2 , glabrous \\
\hline
\end{tabular}

September. Fruiting September-December.

Etymology.- The specific name refers to its similarity to Uraria acuminata.

Notes.-Uraria pseudoacuminata is superficially very similar to $U$. acuminata Kurz, a common species in mainland S.E. Asia. It can be distinguished easily by its glabrous pod which has 2 segments, each with 1 ovule, and pedicels that have both multicellular, glandular hairs and hooked hairs, but rarely straight hairs, on the lower part (Table 1).

Specimens examined. - NORTH-EASTERN : Udon Thani [Nong Saeng, W. Sidajium 54 (BKF)], Bueng Khan [Phu Wua Wild Life Sanctuary, I.C. Nielsen, C. Niyomdham, T. Jonganulak, N. Hemrath \& J. Rithipheth 1580 (AAU), W. Tokaew \& P. Chantaranothai 403 (KKU)], Khonkaen [Phu Khieo, G. Murata, C. Phengklai, S. Mitsuta, H. Nagamasu \& N. Namtasan T-41860 (BKF)]; EASTERN: Chaiyaphum [C.F. van Beusekom, C. Phengklai, R. Geesink \& B. Wongwan 9167 (BKF)], Nakhon Ratchasima [Sakae Rat, C.F. van Beusekom, Chan Wid \& R. Geesink 3299 (BKF, C, K), S.N. 374 (BKF 46328)]; SOUTHEASTERN: Chanthaburi [Pongnamron, B. Sangkhachand s.n. (BKF 13174)]. 
Acknowledgements: We would like to thank the Thailand Research Fund and National Centre for Genetic Engineering and Biotechnology, grant BRT R 150008 for financial support. We also thank to the curators and staff of $\mathrm{AAU}, \mathrm{ABD}, \mathrm{BCU}, \mathrm{BK}, \mathrm{BKF}, \mathrm{C}, \mathrm{CMU}, \mathrm{K}, \mathrm{KKU}, \mathrm{TCD}$, PSU, QBG, and Herbarium of the Department of Biology, Chiang Mai University for their facilities.

\section{REFERENCES}

1. Ohashi H (2005) Tribe Desmodieae. In: Lewis G, Schrire B, Mackinder B, Lock M (eds) Legumes of the World, The Royal Botanic Gardens, Kew, pp 433-45.

2. Craib WG, Kerr AFG (1932) Florae Siamensis Enumeratio, a List of the Plants Known from Siam with Records of their Occurrence, Vol. 2. Siam Society, Bangkok.

3. Thiers B (2012) Index Herbariorum: A global directory of public herbaria and associated staff, New York Botanical Garden's Virtual Herbarium. http://sweetgum.nybg. org/ih/. 